\title{
EUCLIDEAN NETWORKS WITH A BACKBONE AND A LIMIT THEOREM FOR MINIMUM SPANNING CATERPILLARS
}

\author{
PETAR JEVTIĆ AND J. MICHAEL STEELE
}

\begin{abstract}
A caterpillar network (or graph) $G$ is a tree with the property that removal of the leaf edges of $G$ leaves one with a path. Here we focus on minimum weight spanning caterpillars (MSCs) where the vertices are points in the Euclidean plane and the costs of the path edges and the leaf edges are multiples of their corresponding Euclidean lengths. The flexibility in choosing the weight for path edges versus the weight for leaf edges gives some useful flexibility in modeling. In particular, one can accommodate problems motivated by communications theory such as the "last mile problem." Geometric and probabilistic inequalities are developed that lead to a limit theorem that is analogous to the well-known Beardwood, Halton Hammersley theorem for the length of the shortest tour through a random sample.

KEY WORDS: Euclidean networks, caterpillar graphs, shortest paths, traveling salesman problem, Beardwood, Halton, Hammersley theorem, minimal spanning trees, Gutman graphs.

Mathematics Subject Classification (2000): Primary: 60C05, 90C40; Secondary: 60G42, 90C27, 90C39
\end{abstract}

\section{INTRODUCTION}

Given a set $\chi=\left\{x_{1}, x_{2}, \ldots, x_{n}\right\}$ of $n$ points in $\mathbb{R}^{2}$, the set $\mathcal{C}=\mathcal{C}(\chi)$ of spanning caterpillars for $\chi$ is the set of all connected graphs $G$ such that $G$ has vertex set $\chi$ and there exists a path $\pi$ of edges in $G$ such that if the edges of $\pi$ are removed then the remaining subgraph is a collection of one or more disjoint stars. Here, as usual, a star is simply a connected graph with at most one vertex that has degree greater than one. To visualize the typical picture, just draw a long path graph and add a liberal sprinkling of edges incident to the vertices on the path.

The main focus here is on minimum weighted spanning caterpillars where we differentiate between the costs of edges that are on the path and those that are not on the path. To make this precise, we first note that the full specification of a spanning caterpillar requires three elements. One has to specify the vertex set $\chi$, the edge set $E$, and some path $\pi$ in $G$ such that if the edges of $\pi$ are removed from $E$ one is left with a collection of disjoint stars with vertex set $\chi$. Typically there are multiple options for the path $\pi$, so a fully specified spanning caterpillar $G$ is given by a triple $G=(\chi, E, \pi)$.

For each edge $e$ in the edge set of $G$, we let $|e|$ denote its Euclidean length; that is, if $e=(x, y) \in E=E(G)$ then we have $|e|=|x-y|$. Now, given a fixed $\lambda>0$,

P. Jevtić: University of Torino, Torino, Italy.

J. M. Steele: Wharton School, Department of Statistics, Huntsman Hall 447, University of Pennsylvania, Philadelphia, PA 19104. 
we define the weight $W(G)$ of the spanning caterpillar $G=(\chi, E, \pi)$ to be

$$
W(G)=\lambda \sum_{e \in \pi}|e|+\sum_{e \in \pi^{c}}|e|
$$

One motivation for this weighting scheme is the infamous "last mile" problem of communication network theory. In that context, the weight factor $\lambda$ for the path edges would be smaller than one - possibly much smaller since communication along a network backbone may be very fast. Nevertheless, there is no mathematical reason to restrict the value of $\lambda$ beyond requiring it to be positive; moreover, there are benefits to being flexible about the size of $\lambda$. For example, in a ground transportation model where the "drop off" cost is cheap, one would want to take $\lambda$ bigger than one.

Here we are concerned with the cost of the minimum weight spanning caterpillar under two situations. First, there is instructive geometry to be understood when the points are placed deterministically — or even adversatively. Second, one wants to understand the generic situation - say when the set $\chi_{n}$ is given by $\left\{X_{1}, X_{2}, \ldots, X_{n}\right\}$ and the random points $X_{i}, 1 \leq i \leq n$ are independent and identically distributed in $\mathbb{R}^{2}$. In this generic scenario, the random variable of primary interest is the weight of the minimum spanning caterpillar of the random sample $\chi_{n}$ :

$$
M\left(\boldsymbol{\chi}_{n}\right)=M\left(X_{1}, X_{2}, \ldots, X_{n}\right) \stackrel{\text { def }}{=} \min \left\{W(G): G \in \mathcal{C}\left(\boldsymbol{\chi}_{n}\right)\right\} .
$$

Our main theorem is a strong law for $M\left(X_{1}, X_{2}, \ldots, X_{n}\right)$ that is of a kind that goes back to Beardwood, Halton and Hammersley (1959) for the traveling salesman problem.

Theorem 1 (Strong Law for MSCs of Random Samples from the Square). If the random points $X_{i}, i=1,2, \ldots$ are chosen independent and uniformly from the unit square, then there is a constant $\beta_{M S C}(\lambda)>0$ such that

$$
\lim _{n \rightarrow \infty} n^{-1 / 2} M\left(X_{1}, X_{2}, \ldots, X_{n}\right)=\beta_{M S C}(\lambda) \quad \text { with probability one. }
$$

More generally, if the random variables $X_{i}, i=1,2, \ldots$ are independent and have a density $f$ on $\mathbb{R}^{2}$ that has compact support, then we have with probability one that

$$
\lim _{n \rightarrow \infty} n^{-1 / 2} M\left(X_{1}, X_{2}, \ldots, X_{n}\right)=\beta_{M S C}(\lambda) \int_{\mathbb{R}^{2}} \sqrt{f(x)} d x,
$$

where the constant $\beta_{M S C}(\lambda)$ in (3) is the same as in (2).

Small values of $\lambda$ favor path edges over leaf edges, so it is natural to ask if Theorem 1 might actually be a generalization of the Beardwood, Halton, Hammersley theorem. As the theorem is framed and proved it does not rigorously include the that theorem. Nevertheless, one can give a theorem that covers both the behavior of minimum spanning caterpillars and the minimum cost traveling salesman paths. We will return to this point in later sections where we also discuss the relationship between the MSC constant $\beta_{M S C}(\lambda)$ and the corresponding constants $\beta_{T S P}$ and $\beta_{M S T}$ for the traveling salesman problem and the minimum spanning tree problem.

Limit results like the Beardwood, Halton, Hammersley theorem have an extensive literature, much of which is summarized in the monographs of Yukich (1998) and Steele (1997). Nevertheless, the development here is specifically attentive to minimal spanning caterpillars, and it is largely independent of the general theory. 
Section 2 develops the key geometric facts that one needs to prove our theorem. Subsequent sections complete the proof and discuss applications of the theorem.

\section{Geometric Features of Minimum Spanning Caterpillars}

Several of our inferences about the structure of a minimal spanning caterpillar will depend on estimates of the weight of a suboptimal spanning caterpillar. Some of these depend in turn on a classic bound for the length of the shortest path through a set of points in a square.

Lemma 1 (Short Path Bound). For any $\left\{y_{1}, y_{2}, \ldots, y_{m}\right\} \subset[0, t]^{2}, t>0$, there is a permutation $\sigma:[1, m] \rightarrow[1, m]$ such that

$$
\sum_{i=1}^{m-1}\left|y_{\sigma(i)}-y_{\sigma(i+1)}\right| \leq 3 t \sqrt{m} .
$$

In other words, given a vertex set $\left\{y_{1}, y_{2}, \ldots, y_{m}\right\}$ in a square of side $t$, we can always find a path that is not longer than $3 t \sqrt{m}$. Results of Few (1955) are more precise (and still easily proved). For our purposes here, any explicit $O(t \sqrt{m})$ bound would suffice.

One of the features that makes the minimum spanning caterpillar problem challenging is that the cost can go up or down as one adds points. For example, if $\chi=\left\{x_{1}, x_{2}, x_{3}, x_{4}\right\}$ is the set of corner points of the square $[0,1]^{2}$, then with $\lambda=1$ we have $M(\chi)=3$, but if $x_{5}=\left(2^{-1 / 2}, 2^{-1 / 2}\right)$ and $\chi^{\prime}=\chi \cup\left\{x_{5}\right\}$ then $M\left(\chi^{\prime}\right)=2^{3 / 2}<3$. In particular, $M(\cdot)$ is not a Subadditive Euclidean functional in the sense of Steele (1997). The tools of Section 2 help us to deal with this and related geometric difficulties.

Several of our arguments depend on decompositions of the unit square into subsquares, and the most central of these is given by the next lemma. Here by $\mathcal{B}(k)$ we denote the collection of all of the $k^{2}$ subsquares of $[0,1]^{2}$ that have the form

$$
[a / k,(a+1) / k] \times[b / k,(b+1) / k], \quad \text { where } 0 \leq a, b<k .
$$

For any graph $G$ and any vertex $y$ of $G$ we let $N_{G}(y)$ be the set of the neighbors of $y$ in $G$. Also to any $y \in \mathbb{R}^{2}$ we associate a family of annuli

$$
A(y, r)=\left\{x \in \mathbb{R}^{2}: r / 2 \leq|x-y| \leq r\right\} \quad 0 \leq r<\infty .
$$

Lemma 2 (No Crowded Annulus). There exists a constant $\alpha=\alpha(\lambda)>0$ such that for any set $\chi=\left\{x_{1}, x_{2}, \ldots, x_{n}\right\} \subset[0,1]^{2}$ and for any minimum spanning caterpillar $G=(\chi, E, \pi)$ we have for all $y \in \chi$ and all $r>0$ that

$$
\left|N_{G}(y) \cap A(y, r)\right| \leq \alpha .
$$

Proof. If $y$ is a star point of $G$ the assertion is trivial since $y$ has just one neighbor. Hence we assume that $y \in \pi$ and — for the moment - we further assume that $y$ is an interior vertex of $\pi$ with neighbors $y_{1}$ and $y_{2}$ on $\pi$ as show in Figure 1 . We now let $m=\left|N_{G}(y) \cap A(y, r)\right|$ and we assume with out loss that $m \geq 4$. We will now construct a new spanning graph $G^{\prime}$ of $\chi$, as shown in Figure 2 , and use the suboptimality of $G^{\prime}$ to get a bound on $m$.

First, delete all of the edges from $y$ to the star points $S$ of $G$ in $N_{G}(y) \cap A(y, r)$. Thus, we delete at least $m-2$ edges with a total cost at least $(m-2) r / 2$. Next we apply Lemma 1 to get a path $\pi_{0}$ trough the points of $S$ such that the Euclidean length of $\pi_{0}$ is not greater than $6 \mathrm{rm}^{1 / 2}$; here we use the observation that the annulus 


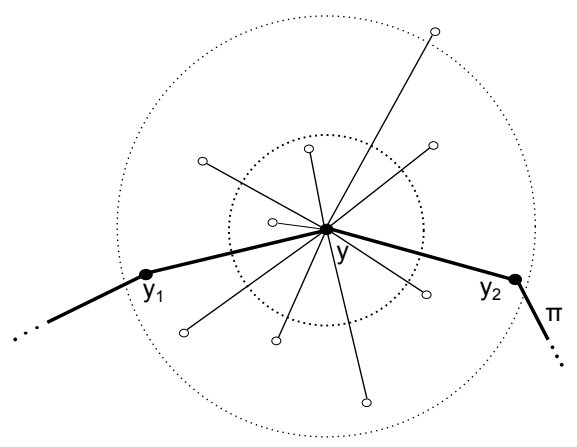

Figure 1. The annulus with the inner radius $r / 2$ and the outer radius $r$ centered at the path point $y$. Open dots denote non-path points of $G$ and the heavy line indicates the path $\pi$.

is contained in a box with side $2 r$ and $m$ is an upper bound on the number of points in $S$. We let $z_{1}$ and $z_{2}$ be the endpoints of the path $\pi_{0}$. These are distinct by our assumption that $m \geq 4$.

To complete the construction, we add the edge $\left(y, z_{1}\right)$, delete the edge $\left(y, y_{2}\right)$ and insert the edge $\left(z_{2}, y_{2}\right)$. Consequently, for the path $\pi^{\prime}$ for $G^{\prime}=\left(\chi, E^{\prime}, \pi^{\prime}\right)$ we can take the segment of $\pi$ up to $y$, the edge $\left(y, z_{1}\right)$, the path $\pi_{0}$ through $S$ from $z_{1}$ to $z_{2}$, the edge $\left(z_{2}, y_{2}\right)$ and then finally we take the remainder of the original path $\pi$ that follows $y_{2}$.

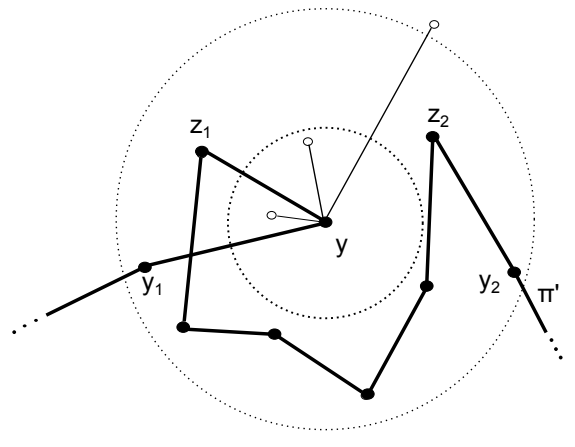

Figure 2. A view of the annulus of Figure 1 after surgery. In the new caterpillar $G^{\prime}$, all points of $\chi$ that are in the annulus are now on the new path $\pi^{\prime}$.

By our construction we have

$W\left(G^{\prime}\right) \leq W(G)-(m-2) r / 2+6 \lambda r m^{1 / 2}+\lambda\left|y-z_{1}\right|-\lambda\left|y-y_{2}\right|+\lambda\left|z_{2}-y_{2}\right|$.

By the triangle inequality we have $\left|z_{2}-y_{2}\right|-\left|y-y_{2}\right| \leq r$ and trivially $\left|y-z_{1}\right| \leq r$, so from $W(G) \leq W\left(G^{\prime}\right)$ we have

$$
m \leq 12 \lambda m^{1 / 2}+4 \lambda+2 .
$$

Therefore, for case when $y$ is an interior point of $\pi$ we can take the generous bound $m \leq(14 \lambda+2)^{2}$. The case when $y$ is an end point of $\pi$ is completely analogous even a bit easier, so we omit the details for that case. 
A basic consequence of the "No Crowded Annulus" lemma is that a vertex $v$ of a MSC with a large number of neighbors must have some neighbor that is very close to $v$; in fact, it must be exponentially close.

Lemma 3 (Existence of an Exponentially Near Neighbor). There exists constants $C>0$ and $0<\rho<1$ depending only on $\lambda$ such that for any $\chi=\left\{x_{1}, x_{2}, \ldots, x_{n}\right\}$, any spanning caterpillar $G=(\chi, E, \pi), y_{0} \in \chi$, and $R \geq 0$, we have

$$
\min \left\{\left|y-y_{0}\right|: y \in N_{G}\left(y_{0}\right),\left|y-y_{0}\right| \leq R\right\} \leq C \rho^{q} R,
$$

where $q=\left|N_{G}\left(y_{0}\right) \cap\left\{y:\left|y-y_{0}\right| \leq R\right\}\right|$.

Proof. The infinite set of annuli $A\left(y_{0}, R\right), A\left(y_{0}, R 2^{-1}\right), \ldots, A\left(y_{0}, R 2^{-k}\right), \ldots$ cover the punctured disk $\left\{y: 0<\left|y-y_{0}\right| \leq R\right\}$, and by Lemma 2 none of these annuli can contain more that $\alpha$ points of $N_{G}\left(y_{0}\right)$. Let $k$ be the maximal integer for which it holds that $q \geq k \alpha$. Then, one of the annuli $A\left(y_{0}, R 2^{-j}\right)$, with $j \geq k$ must contain a point of $N_{G}\left(y_{0}\right)$; that is,

$$
q \geq k \alpha \quad \text { implies } \min \left\{\left|y-y_{0}\right|: y \in N_{G}\left(y_{0}\right)\right\} \leq 2^{-k} R .
$$

and this is more than is needed for the lemma. In particular, by review of the proof one can check that $C=2$ and $\rho=2^{-1 / \alpha}$ would suffice.

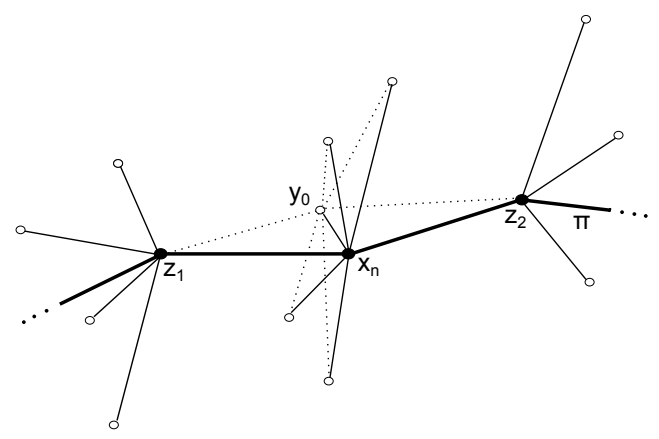

Figure 3 . The caterpillar of Lemma 4 where dotted lines show the new caterpillar after $x_{n}$ is dropped and $y_{0}$ is promoted to become a path vertex. None of the old edges incident to $x_{n}$ are present in the new caterpillar.

Lemma 4 (Cost to Drop One). There is a constant $C=C(\lambda)$ such that for all $\chi=\left\{x_{1}, x_{2}, \ldots, x_{n}\right\}$ and all minimal spanning caterpillars $G=(\chi, E, \pi)$, we have

$$
M\left(x_{1}, x_{2}, \ldots, x_{n-1}\right) \leq M\left(x_{1}, x_{2}, \ldots, x_{n}\right)+C / k,
$$

provided that every $B \in \mathcal{B}(k)$ contains a vertex of the path $\pi$.

Proof. We first set $\chi^{\prime}=\left\{x_{1}, x_{2}, \ldots, x_{n-1}\right\}$. If there is a minimum spanning caterpillar $G=(\chi, E, \pi)$ of $\chi$ where the point $x_{n}$ is not a vertex of the path $\pi=\pi(G)$, then we can simply delete $x_{n}$ and the edge incident to $x_{n}$ to get a minimum spanning caterpillar of $\chi^{\prime}$ that has weight less than $M\left(x_{1}, x_{2}, \ldots, x_{n}\right)$. Similarly, if $x_{n} \in \pi$ but $x_{n}$ has degree one in $G$, then we can just delete $x_{n}$ and its edge to get a spanning caterpillar that has weight less than $M\left(x_{1}, x_{2}, \ldots, x_{n}\right)$. Also, if $x_{n}$ is on $\pi$ and has degree equal to two, then we can delete $x_{n}$ and the edges incident to $x_{n}$ 
and add an edge connecting the neighbors of $x_{n}$ on $\pi$. In all of these easy cases we get a spanning caterpillar for $\chi^{\prime}$ that has weight less than $M(\chi)$.

Thus, we may assume that $x_{n}$ is a vertex of $\pi$ and that $x_{n}$ has at least one neighbor in $G$ that is not on the path $\pi$. As before, we have two cases to consider: (a) when $x_{n}$ is an end point of the path $\pi$ and (b) when $x_{n}$ is an interior point of $\pi$ as shown in Figure 3. The cases are similar, so we will only give the details for the second case.

Setting $m=\left|N_{G}\left(x_{n}\right)\right|$ we have $m \geq 3$ and there is at least one star vertex adjacent to $x_{n}$. Let $\mu$ be smallest distance from $x_{n}$ to a star vertex of $G$ in $N_{G}\left(x_{n}\right)$, and let $y_{0}$ be a star vertex in $N_{G}\left(x_{n}\right)$ with $\mu=\left|y_{0}-x_{n}\right|$. We also let $z_{1}$ and $z_{2}$ be the neighbors of $x_{n}$ on the path $\pi$.

Now we construct a new spanning caterpillar $G^{\prime}=\left(\chi^{\prime}, E^{\prime}, \pi^{\prime}\right)$. To define $E^{\prime}$ we take (a) all of the edges of $E$ not incident to $x_{n}$ and (b) as new edges we add all of the edges $\left(y_{0}, w\right)$ where $w \in N_{G}\left(x_{n}\right) \backslash\left\{y_{0}\right\}$. Since the set $E^{\prime}$ contains the edges $\left(z_{1}, y_{0}\right)$ and $\left(y_{0}, z_{2}\right)$, we define the path $\pi^{\prime}$ of the new spanning caterpillar $G^{\prime}$ by taking the old path $\pi$ up to the vertex $z_{1}$, followed by the edge $\left(z_{1}, y_{0}\right),\left(y_{0}, z_{2}\right)$ and then we follow the old path from $z_{2}$ to the end of $\pi$. This construction is illustrated by Figure 3 .

To estimate the weight $W\left(G^{\prime}\right)$ of the spanning caterpillar that we have constructed, we repeat the construction with bookkeeping. By the triangle inequality and the definitions of $m$ and $\mu$, we have

$$
\begin{aligned}
W\left(G^{\prime}\right) \leq & M\left(x_{1}, x_{2}, \ldots, x_{n}\right)-\lambda\left|z_{1}-x_{n}\right|-\lambda\left|z_{2}-x_{n}\right| \\
& +\lambda\left\{\left|z_{1}-x_{n}\right|+\left|x_{n}-y_{0}\right|\right\}+\lambda\left\{\left|z_{2}-x_{n}\right|+\left|x_{n}-y_{0}\right|\right\} \\
& +(m-3)\left|y_{0}-x_{n}\right| \\
& =M\left(x_{1}, x_{2}, \ldots, x_{n}\right)+(m-3+2 \lambda) \mu .
\end{aligned}
$$

The task now is to bound the last summand, and plan is to exploit Lemma 3 which tells us that if $m$ is large then $\mu$ must be small. We assume that each $B \in \mathcal{B}(k)$ contains a vertex of the path $\pi=\pi(G)$, so the optimality of $G$ implies that the star edges incident to $x_{n}$ cannot have length greater than $R=2^{1 / 2} / k$. This gives us the lower bound

$$
q \equiv\left|N_{G}\left(x_{n}\right) \cap\left\{y:\left|y-x_{n}\right| \leq R\right\}\right| \geq m-2,
$$

so, using Lemma 3 with $R=2^{1 / 2} / k$ gives the bound $\mu \leq C \rho^{m-2} 2^{1 / 2} / k$. Thus, we can generously bound last summand of $(6)$ by

$$
m \mu+2 \lambda \mu \leq C \rho^{-2} 2^{1 / 2} \max _{m}\left\{m \rho^{m}\right\} / k+2 \lambda 2^{1 / 2} / k=O_{\lambda}(1 / k) .
$$

which is all we need.

Lemma 5 (Cost to Add One). There is a constant $C=C(\lambda)$ such that for all $\chi=\left\{x_{1}, x_{2}, \ldots, x_{n-1}\right\}$ and all minimal spanning caterpillars $G=(\chi, E, \pi)$, we have

$$
M\left(x_{1}, x_{2}, \ldots, x_{n}\right) \leq M\left(x_{1}, x_{2}, \ldots, x_{n-1}\right)+C / k,
$$

provided that every $B \in \mathcal{B}(k)$ contains a vertex of the path $\pi$.

Proof. Unlike Lemma 4, this lemma is trivial. To get a spanning caterpillar of $\chi^{\prime}=\chi \cup\left\{x_{n}\right\}$ we just join $x_{n}$ to the nearest path point of the spanning caterpillar 
$G=(\chi, E, \pi)$. If $x_{n} \in B \in \mathcal{B}(k)$ there is a path point $x^{\prime}$ of $G$ in $B$ and we can joint $x^{\prime}$ to $x_{n}$ at a cost not greater than $2^{1 / 2} / k$.

\section{Stochastic Features of the MSC's Backbone}

When the sample $\boldsymbol{\chi}_{n}=\left\{X_{1}, X_{2}, \ldots, X_{n}\right\}$ is independent with the uniform distribution on $[0,1]^{2}$, the minimal spanning caterpillar of $\chi_{n}$ is unique with probability one, and it will be denoted by $G=\left(\chi_{n}, E, \pi\right)$. The path $\pi=\pi(G)$ of the minimal spanning caterpillar is itself a graph, and we denote the vertices of $\pi$ by $\pi_{V}\left(\chi_{n}\right)$. The set vertices of $G$ that are not on the path will be denoted by $\pi_{V}^{c}\left(\chi_{n}\right)$, and the elements of this set are called star points. Every element of $\chi_{n}$ is thus either a star point or a path point.

Lemma 6 (Path Points in the Box). There are two constants $\alpha=\alpha(\lambda)>0$ and $C=C(\lambda)$ such that for all $n, k$ and $B \in \mathcal{B}(k)$ we have

$$
\mathrm{P}\left(\pi_{V}\left(\boldsymbol{\chi}_{n}\right) \cap B=\emptyset\right) \leq C k \exp \left(-\alpha n / k^{3}\right) .
$$

Proof. Let $(G, E, \pi)$ be the minimum spanning caterpillar with vertex set $\chi_{n}$; we observed earlier that for a uniform independent sample, the minimum spanning caterpillar is unique with probability one. To begin, we define $\ell=\ell(k)$ by setting

$$
\ell=\lceil 3(\lambda+\sqrt{k \lambda})\rceil+1 .
$$

Now, for a given box $B \in \mathcal{B}(k)$, we let $\mathcal{H}(\ell, B)$ denote the set of $\ell^{2}$ squares of $\mathcal{B}(3 k \ell)$ that are the middle ninth of $B$; explicitly, $\mathcal{H}(\ell, B)$ is the set of all squares $B^{\prime} \in \mathcal{B}(3 k \ell)$ for which we have

$$
B^{\prime} \subset[a / k+1 /(3 k), a / k+2 /(3 k)] \times[b / k+1 /(3 k), b / k+2 /(3 k)] .
$$

Now we consider the two events

$$
A=\left\{\omega: \pi_{V}\left(\chi_{n}\right) \cap B=\emptyset\right\} \quad \text { and } \quad F=\left\{\omega: \min _{S \in \mathcal{H}(\ell, B)}\left|S \cap \chi_{n}\right|>0\right\} ;
$$

that is, $A$ is the event that there are no path points in the box $B$ and $F$ is the event that each subbox $S \in \mathcal{H}(\ell, B)$ contains at least one point of the vertex set $\chi_{n}$.

If $A \cap F \neq \emptyset$, we take an $\omega \in A \cap F$ and then for $\chi_{n}=\chi_{n}(\omega)$ we construct a new spanning caterpillar $G^{\prime}=\left(\chi_{n}, E^{\prime}, \pi^{\prime}\right)$ as follows:

(1) Since $\omega \in F$ we have $\chi_{n} \cap S \neq \emptyset$ for each of the $\ell^{2}$ subsquares $S \in \mathcal{H}(\ell, B)$ and we select one point $v_{S} \in \chi_{n} \cap S$ for each $S \in \mathcal{H}(\ell, B)$.

(2) We let $\pi_{0}$ be a path through the set of $\ell^{2}$ points $\left\{v_{S}: S \in \mathcal{H}(\ell, B)\right\}$ that is of minimal Euclidean length.

(3) Since $\omega \in A$, no vertices of $\pi_{V}\left(\chi_{n}\right)$ are in $B$, so each element of the set $\left\{v_{S}: S \in \mathcal{H}(\ell, B)\right\}$ is a star point of $G$ and each such $v_{S}$ is connected to path point of caterpillar $G$ that is in $B^{c}$. We call this edge $e_{S}$ and we note that $\left|e_{S}\right| \geq 1 /(3 k)$ since the distance from a point of $S \in \mathcal{H}(\ell, B)$ to a point of $B^{c}$ is at least $1 /(3 k)$.

(4) To define the edge set $E^{\prime}$, we first take the edge set $E$ and remove from $E$ all of the set of edges $\left\{e_{S}: S \in \mathcal{H}(\ell, B)\right\}$. We then add to $E^{\prime}$ the edges of the path $\pi_{0}$ from Step (2). Lastly, we add an edge $e^{\prime}$ that connects end point of $\pi_{0}$ to an end point of $\pi$. It does not matter how one makes the last choice from the four possibilities. The only control over the length of $e^{\prime}$ is that $\left|e^{\prime}\right| \leq 2^{1 / 2}$. 
(5) To complete the specification of the spanning graph $G^{\prime}=\left(\chi_{n}, E^{\prime}, \pi^{\prime}\right)$, we take $\pi^{\prime}$ to be the path consisting of the edges of the old path $\pi$, the connecting edge $e^{\prime}$, and the path $\pi_{0}$ from Step (2).

To estimate the weight of $G^{\prime}$ we recall $\left|e_{S}\right| \geq 1 /(3 k)$, bound the length of $\pi_{0}$ by Lemma 1 (with $t=1 /(3 k)$ ), and use the generous bound $\left|e^{\prime}\right| \leq 2$ to get

$$
\begin{aligned}
W\left(G^{\prime}\right) & \leq W(G)-\sum_{S \in \mathcal{H}(\ell, B)}\left|e_{S}\right|+\lambda \sum_{e \in \pi_{0}}|e|+\lambda\left|e^{\prime}\right| \\
& \leq W(G)-\ell^{2} /(3 k)+\ell \lambda / k+2 \lambda .
\end{aligned}
$$

By the minimality of the weight of the spanning caterpillar $G$ we have that $W(G) \leq W\left(G^{\prime}\right)$, so by solving a quadratic equation we see that the bound 10 . implies that

$$
\ell \leq 3(\lambda+\sqrt{k \lambda}) .
$$

By our choice (8) of $\ell=\ell(k)$, the bound (11) does not hold, so we conclude that $A \cap F=\emptyset$. have

Consequently, we have $A \subset F^{c}$ and since there are $\ell^{2}$ elements of $\mathcal{H}(\ell, B)$ we

$$
\mathrm{P}(A) \leq \mathrm{P}\left(F^{c}\right) \leq \ell^{2}\left(1-\frac{1}{9 \ell^{2} k^{2}}\right)^{n} \leq \ell^{2} \exp \left(-\frac{n}{9 \ell^{2} k^{2}}\right) .
$$

Again, using the specification given by $(8)$ of $\ell=\ell(k)=O(\sqrt{k})$, we have

$$
0<\inf _{k \geq 1}\left\{\frac{k}{9 \ell^{2}(k)}\right\}
$$

and we then take $\alpha=\alpha(\lambda)$ to be any constant less than this infimum. Again we observe by our choice (8) we have $\ell^{2}=O(k)$, so we can then choose $C=C(\lambda)$ such that we have the bound (7) for all $n \geq 1$ and $k \geq 1$.

\section{Moments of Change-One Bounds and the Variance}

Lemma 7 (Expected Cost of Change). For all $1 \leq p<\infty$ and all $\epsilon>0$ we have the bound

$$
\mathrm{E}\left[\left|M\left(\chi_{n+1}\right)-M\left(\chi_{n}\right)\right|^{p}\right]=O_{\lambda, p, \epsilon}\left(n^{\epsilon-p / 3}\right) \quad \text { for } n \rightarrow \infty .
$$

Proof. First we fix $k$ and consider the set

$$
F_{n}(k)=\left\{\omega: B \cap \pi_{V}\left(\chi_{n}\right) \neq \emptyset \text { for all } B \in \mathcal{B}(k)\right\}
$$

Since there are $k^{2}$ elements of $\mathcal{B}(k)$, Boole's inequality and Lemma 6 give us a bound on the complementary event,

$$
P\left(F_{n}^{c}(k)\right) \leq C k^{3} \exp \left(-\alpha n / k^{3}\right) .
$$

and for $F_{n-1}^{c}(k)$ we have the analogous bound.

Now, by using Lemmas 4 and 5 for $\omega \in F_{n}(k) \cap F_{n-1}(k)$ and applying Lemma 1 for $\omega \in F_{n}^{c}(k) \cup F_{n-1}^{c}(k)$ we have the pointwise bound

$$
\left|M\left(\chi_{n+1}\right)-M\left(\chi_{n}\right)\right| \leq C / k+C n^{1 / 2} \mathbb{1}\left[F_{n}^{c}(k) \cup F_{n-1}^{c}(k)\right] .
$$


Taking $p$ 'th powers and using $(a+b)^{p} \leq 2^{p}\left(a^{p}+b^{p}\right)$ on the right side of (16), we find from the bound 15 that for a new constant $C=C(p)$ we have

$$
E\left[\left|M\left(\chi_{n+1}\right)-M\left(\chi_{n}\right)\right|^{p}\right] \leq C / k^{p}+C n^{p / 2} k^{3} \exp \left(-\alpha n / k^{3}\right) .
$$

Finally, taking $k=\left\lfloor n^{1 / 3-\epsilon / p}\right\rfloor$ gives us 13 .

There is a general inequality for the variance that works nicely with "discrete continuity" like that provided Lemma 7. To state the inequality, first consider a set of $2 n$ independent random variables $\left\{X_{i}, X_{i}^{\prime}: 1 \leq i \leq n\right\}$ that take values in $S=\mathbb{R}^{d}$. Next, given a Borel function $f: S^{n} \rightarrow \mathbb{R}$ we set $F=f\left(X_{1}, X_{2}, \ldots, X_{n}\right)$ and for $1 \leq i \leq n$ we set

$$
F_{i}=f\left(X_{1}, \ldots, X_{i-1}, X_{i}^{\prime}, X_{i+1}, \ldots, X_{n}\right) .
$$

One then has the jackknife bound (see e.g. Steele (1986) or Boucheron, Lugosi and Bousquet (2004)):

$$
\operatorname{Var} F \leq \frac{1}{2} \mathrm{E} \sum_{i=1}^{n}\left(F-F_{i}\right)^{2} .
$$

From this inequality and the estimate 13 with $p=2$, one immediately gets a useful bound on the variance of $M\left(\chi_{n}\right)$.

Lemma 8 (Variance Estimate). For all $\epsilon>0$ we have

$$
\operatorname{Var} M\left(\chi_{n}\right)=O_{\lambda, \epsilon}\left(n^{\epsilon+1 / 3}\right) .
$$

With the variance of $M\left(\chi_{n}\right)$ under control, the proof of Theorem 1 will be in range once we determine the asymptotic behavior of $E M\left(\chi_{n}\right)$.

\section{Asymptotics of the Mean}

Let $\{N(t): 0 \leq t<\infty\}$ be a standard Poisson process with arrival rate one, and let $\left\{X_{1}, X_{2}, \ldots\right\}$ be an independent sequence of random vectors with the uniform distribution on $[0,1]^{2}$. The sequence is assumed to be independent of the process $\{N(t): 0 \leq t<\infty\}$. Next we set

$$
\chi_{N(t)}=\left\{X_{1}, X_{2}, \ldots, X_{N(t)}\right\} \quad \text { and } \quad \varphi(t)=\mathrm{E}\left[M\left(\chi_{N(t)}\right)\right] .
$$

The idea behind this construction is that $\varphi(t)$ is a smoothed version of the sequence of expected means, and we have the added benefit that for each $B \in \mathcal{B}(k)$ the cardinality of the set $\left\{X_{1}, X_{2}, \ldots, X_{N(t)}\right\} \cap B$ is Poisson with mean $t / k^{2}$. This observation leads to a simple inequality from which we can deduce the asymptotic behavior of $\varphi(t)$.

Lemma 9 (Poisson Averages of the Means). For all $t>0$, and all integer $k>0$ we have

$$
\varphi(t) \leq k \varphi\left(t / k^{2}\right)+3 k \lambda,
$$

and there is a constant $\beta_{M S C}=\beta_{M S C}(\lambda)>0$ such that

$$
\lim _{t \rightarrow \infty} \varphi(t) / \sqrt{t}=\beta_{M S C} .
$$


Proof. For each $B \in \mathcal{B}(k)$, we let $S_{B}=B \cap\left\{X_{1}, X_{2}, \ldots, X_{N(t)}\right\}$. We then let $G_{B}=\left(S_{B}, E_{B}, \pi_{B}\right)$ be the unique minimum spanning caterpillar for $S_{B}$. Assume for the moment there is at least one edge of $\pi_{B}$ for all $B$ and let $a_{B}$ and $b_{B}$ be the two distinct end points of $\pi_{B}$. We then sew together all the paths $\pi_{B}, B \in \mathcal{B}(k)$. Specifically, we order the boxes of $\mathcal{B}(k)$ in Boustrophedon (or plowman's) order; that is, we start on the upper left, go right along the top row, move down to the second row, then move left along the second row, etc. We connect $b_{B}$ to $a_{B^{\prime}}$ where $B^{\prime}$ is a successor of $B$. The Euclidean length of this stitching can be bound above (very crudely) by $3 k$. This gives the bound

$$
M\left(X_{1}, X_{2}, \ldots, X_{N(t)}\right) \leq 3 k \lambda+\sum_{B} W\left(G_{B}\right),
$$

To remove the assumption that each $S_{B}$ is not empty, we just note that if for some $B$ we have $S_{B}=\emptyset$ we just can skip that box when we sew the small caterpillars together to get our spanning caterpillar $\chi_{n}$. Similarly, if for some $B$ the caterpillar $G_{B}$ is a star with central vertex $v$ we just take $a_{B}=b_{B}=v$. We can then go ahead with our sewing as before, and the bound 21) again applies. Finally, we take expectations in the bound (21). Scaling by both side and area, gives us $E\left[W\left(G_{B}\right)\right]=\varphi\left(t / k^{2}\right) k^{-1}$, and there are $k^{2}$ summands in sum, so in the end we have 19$)$.

To prove (20), we replace $t$ with $k^{2} t$ in 19 and we divide by $\sqrt{k^{2} t}$ to get the stabilized recursive inequality,

$$
\frac{\varphi\left(k^{2} t\right)}{\sqrt{k^{2} t}} \leq \frac{\varphi(t)}{\sqrt{t}}+\frac{3 \lambda}{\sqrt{t}} \text { for all } 1 \leq k<\infty \text { and } t>0 .
$$

Now, given any $\epsilon>0$ and any $T<\infty$, we can find by the continuity of $\varphi$ an interval $(a, b)$ such that $T<a<b$ and for which we have

$$
\frac{\varphi(t)}{\sqrt{t}} \leq \epsilon+\liminf _{s \rightarrow \infty} \frac{\varphi(s)}{\sqrt{s}} \equiv \epsilon+\gamma \quad \text { for all } t \in(a, b) .
$$

Choosing $T \geq(3 \lambda / \epsilon)^{2}$, we then have by 22 and 23 that

$$
\frac{\varphi\left(k^{2} t\right)}{\sqrt{k^{2} t}} \leq \gamma+2 \epsilon \quad \text { for all } 1 \leq k<\infty \text { and all } t \in(a, b),
$$

or, in other words, we have

$$
\frac{\varphi(t)}{\sqrt{t}} \leq \gamma+2 \epsilon \quad \text { for all } t \in \bigcup_{k=1}^{\infty}\left(k^{2} a, k^{2} b\right) \equiv S .
$$

For $k \geq 3 a(b-a)^{-1}$ the intervals $\left(k^{2} a, k^{2} b\right)$ and $\left((k+1)^{2} a,(k+1)^{2} b\right)$ overlap, so we have $\left(3^{2} a^{2}(b-a)^{-2} a, \infty\right) \subset S$, and consequently we have

$$
\frac{\varphi(t)}{\sqrt{t}} \leq \gamma+2 \epsilon \text { for all } t>3^{2} a^{3}(b-a)^{-2} .
$$

Since $\epsilon>0$ is arbitrary, this is more than we need for the proof.

Now we want to extract the asymptotic behavior of $\operatorname{E~} M\left(\chi_{n}\right)$ from what we have learned about $\varphi(t)$. One could appeal to the Tauberian theory for Borel means (cf. Korevaar (2004, Chapter 6)), but E $M\left(\chi_{n}\right)$ is so well-behaved that it is quicker to use bare hands. 
Lemma 10 (Mean Increments and DePoissonization).

$$
\lim _{n \rightarrow \infty} \operatorname{E} M\left(\chi_{n}\right) / \sqrt{n}=\beta_{M S C}(\lambda)>0 .
$$

Proof. If we set $A(n)=\mathrm{E}\left[M\left(\chi_{n}\right)\right]$ and take $N_{t}$ to be a Poisson random variable with mean $t$, then by conditioning and the definition of $\varphi$ we have

$$
\varphi(t)=\mathrm{E} A\left(N_{t}\right)=\sum_{j=0}^{\infty} A(j) P\left(N_{t}=j\right)=\sum_{j=0}^{\infty} A(j) \frac{t^{j}}{j !} e^{-t} .
$$

Fixing $0<\epsilon<1 / 6$, we then write

$$
t_{+}(\epsilon)=t+t^{1 / 2+\epsilon} \quad \text { and } t_{-}(\epsilon)=t-t^{1 / 2+\epsilon},
$$

and, by repeated applications of 13 with $p=1$, we get the relation

$$
\sup _{j \in\left[t_{-}(\epsilon), t_{+}(\epsilon)\right]} \mid\left(A(j)-A(\lfloor t\rfloor) \mid=O\left(t^{2 \epsilon+1 / 6}\right) .\right.
$$

Now we make estimations over the three ranges. On the mid-range $\left[t_{-}(\epsilon), t_{+}(\epsilon)\right]$ we use (25), and on the outside ranges $\left[0, t_{-}(\epsilon)\right]$, and $\left[t_{+}(\epsilon), \infty\right)$ we use the bound from Lemma 1 that tells us $A(j) \leq C j^{1 / 2}$ for all $j$. Assembling the three pieces we have

$$
\begin{aligned}
|\varphi(t)-A(\lfloor t\rfloor)| & =\sum_{j=0}^{\infty}|\{A(j)-A(\lfloor t\rfloor)\}| e^{-t} t^{j} / j ! \\
& =O\left(t^{1 / 2} P\left(N_{t} \leq t_{-}(\epsilon)\right)\right)+O\left(t^{2 \epsilon+1 / 6}\right)+O\left(E\left(N_{t}^{1 / 2} \mathrm{I}\left(N_{t} \geq t_{+}(\epsilon)\right)\right)\right) \\
& =o\left(t^{1 / 2}\right) .
\end{aligned}
$$

To check this, note that first summand is $o\left(t^{1 / 2}\right)$ because $P\left(N_{t} \leq t_{-}(\epsilon)\right)=o(1)$, and the last summand is $o\left(t^{1 / 2}\right)$ by the Cauchy-Schwarz inequality and the exponential estimate for the upper Poisson tail. From this bound and the limit $(20)$ we have the limit (24). Finally, for the strict positivity of $\beta_{M S C}(\lambda)$, one can look ahead to the comparison of $\beta_{M S C}(\lambda)$ to $\beta_{M S T}$ in the inequality (34) of Section 7 .

\section{Completion of the Argument}

The tools are in place to complete the proof of the first assertion of Theorem 1. We first note that if we set $n_{j}=j^{2}$, then by the variance bound 18 we have

$$
\operatorname{Var}\left[n_{j}^{-1 / 2} M\left(\chi_{n_{j}}\right)\right]=O\left(j^{2 \epsilon-4 / 3}\right) .
$$

Now, taking $0<\epsilon<1 / 6$, Chebyshev's inequality gives us for all $\delta>0$ that

$$
\sum_{j=1}^{\infty} P\left(\left|n_{j}^{-1 / 2}\left(M\left(\chi_{n_{j}}\right)-E M\left(\chi_{n_{j}}\right)\right)\right| \geq \delta\right) \leq \delta^{-2} \sum_{j=1}^{\infty} \operatorname{Var}\left[n_{j}^{-1 / 2} M\left(\chi_{n_{j}}\right)\right]<\infty .
$$

The Borel-Cantelli lemma, Lemma 10, and the arbitrariness of $\delta$ then tell us that

$$
\lim _{j \rightarrow \infty} n_{j}^{-1 / 2} M\left(\chi_{n_{j}}\right)=\beta_{M S C} \quad \text { a.s.. }
$$

Next, fix $k$ and recall the set $F_{n}(k)=\left\{\omega: B \cap \pi_{V}\left(\chi_{n}\right) \neq \emptyset\right.$ for all $\left.B \in \mathcal{B}(k)\right\}$ that was introduced in the proof of Lemma 7. We observed there that we have the bound $P\left(F_{n}^{c}(k)\right) \leq C k^{3} \exp \left(-\alpha n / k^{3}\right)$, so by another application of the BorelCantelli lemma we have $P\left(F_{n}^{c}(k)\right.$ i.o. $)=0$. 
Now, given $n$ we define $j$ by the relations $n_{j} \leq n<n_{j+1}$. We can then apply Lemma 1 for $\omega \in F_{n_{j}}^{c}(k)$ and apply Lemma 5 for $\omega \in F_{n_{j}}(k)$ to get the bound

$$
M\left(\boldsymbol{\chi}_{n}\right) \leq M\left(\boldsymbol{\chi}_{n_{j}}\right)+C\left(n-n_{j}\right) k^{-1}+3 n^{1 / 2} \max (1, \lambda) \mathbb{1}\left(F_{n_{j}}^{c}(k)\right) .
$$

Dividing by $n^{1 / 2}$, taking the limsup, using $(26)$, and recalling the definition of $n_{j}$, we find

$$
\limsup _{n \rightarrow \infty} n^{-1 / 2} M\left(\chi_{n}\right) \leq \beta_{M S C} \quad \text { with probability } 1,
$$

and this proves half of the assertion of Theorem 1 .

With the natural changes, one can prove the second half. This time we apply Lemma 1 for $\omega \in F_{n}^{c}(k)$ and use Lemma 5 for $\omega \in F_{n}(k)$ to get the bound

$$
M\left(\chi_{n_{j+1}}\right) \leq M\left(\chi_{n}\right)+C\left(n_{j+1}-n\right) k^{-1}+3 n_{j+1}^{1 / 2} \max (1, \lambda) \mathbb{1}\left(F_{n}^{c}(k)\right),
$$

so, when we divide by $n^{1 / 2}$ and take the liminf on both sides, we find from 26 . that

$$
\beta_{M S C} \leq \liminf _{n \rightarrow \infty} n^{-1 / 2} M\left(\chi_{n}\right) \quad \text { with probability } 1,
$$

completing the proof of the first assertion of Theorem 1 .

One can prove the second assertion of Theorem 1, by a variation of the approximation argument that Beardwood, Halton and Hammersley (1959) used in analysis of the traveling salesman problem. At this point the argument is routine, so we will just give a sketch.

First, we note that if the random variables $X_{i}, i=1,2, \ldots$ have a density $f$ with compact support in $\mathbb{R}^{2}$, then by translation and scaling we can suppose without loss of generality that the support of $f$ is contained in $[0,1]^{2}$. Next we note that we can approximate $f$ as well as we like by a density $\phi$ that is constant on each of the subsquares $B$ in our decomposition $\mathcal{B}(k)$ of $[0,1]^{2}$ into squares of side $1 / k$. More precisely, for any $\epsilon>0$ there is an integer $k$ and there is a constant $\alpha(B) \geq 0$ for each $B \in \mathcal{B}(k)$ such that the weighted sum of indicator functions

$$
\phi(x)=\sum_{B \in \mathcal{B}(k)} \alpha(B) \mathbb{1}_{B}(x)
$$

is a density on $[0,1]^{2}$ and

$$
\int_{\mathbb{R}^{2}}|f(x)-\phi(x)| d x \leq \epsilon .
$$

Now, by the existence of a maximal coupling (see e.g. Lindvall (1992), p.18), we can choose an independent sequence $Z_{i}=\left(X_{i}, Y_{i}\right), i=1,2, \ldots$ such that for all $i$, $X_{i}$ has density $f, Y_{i}$ has density $\phi$, and

$$
P\left(X_{i} \neq Y_{i}\right) \leq \epsilon .
$$

Since we have $P\left(Y_{i} \in B\right)=\alpha_{B} / k^{2}$, we see by the law of large numbers that

$$
\left|\left\{Y_{1}, Y_{2}, \ldots, Y_{n}\right\} \cap B\right| \sim n \alpha_{B} / k^{2} \quad \text { with probability one, }
$$


so, by scaling and the first part (2) of Theorem 1 we have with probability one for each $B \in \mathcal{B}(k)$ that

$$
\begin{aligned}
\lim _{n \rightarrow \infty} M\left(\left\{Y_{1}, Y_{2}, \ldots, Y_{n}\right\} \cap B\right) / \sqrt{n} & =\frac{1}{k} \beta_{M S C}(\lambda) \sqrt{\alpha_{B} / k^{2}} \\
& =\beta_{M S C}(\lambda) \int_{B} \sqrt{\phi(x)} d x
\end{aligned}
$$

since $\phi(x)=\alpha_{B}$ for all $x \in B$. The union of the minimum spanning caterpillars $\left\{Y_{1}, Y_{2}, \ldots, Y_{n}\right\} \cap B$ over all subsquares $B \in \mathcal{B}(k)$ has a length that differs from the length of the minimum spanning caterpillar of the whole sample $\left\{Y_{1}, Y_{2}, \ldots, Y_{n}\right\}$ by an amount that is bounded independently of $\mathrm{n}$ (for $k$ fixed), so by summing the previous limit we have

$$
\lim _{n \rightarrow \infty} M\left(\left\{Y_{1}, Y_{2}, \ldots, Y_{n}\right\}\right) / \sqrt{n}=\beta_{M S C}(\lambda) \int_{\mathbb{R}^{2}} \sqrt{\phi(x)} d x .
$$

Finally, by the strong law of large numbers and the coupling bound (27) applied to the sum of the indicators $\mathbb{1}\left(X_{i} \neq Y_{i}\right)$, we see that the cardinality of the difference between the sets $\left\{Y_{1}, Y_{2}, \ldots, Y_{n}\right\}$ and $\left\{X_{1}, X_{2}, \ldots, X_{n}\right\}$ is almost surely $O(\epsilon n)$. Consequently we have that the difference between $M\left(Y_{1}, Y_{2}, \ldots, Y_{n}\right)$ and $M\left(X_{1}, X_{2}, \ldots, X_{n}\right)$ is almost surely $O(\sqrt{\epsilon n})$ so by $(29)$ and the arbitrariness of $\epsilon>0$, one has the second conclusion (3) of Theorem 1 .

\section{Connecting the MSC, MSt, and TSP: Constants and Complexity}

The limiting constant $\beta_{M S C}(\lambda)$ of Theorem 1 has a natural relationship to the corresponding limit constants $\beta_{M S T}$ and $\beta_{T S P}$ for the minimal spanning tree problem and the traveling salesman problem. Although the values of $\beta_{M S T}$ and $\beta_{T S P}$ are not known exactly, they have been investigated repeatedly (see e.g. Finch (2003, pp. 497-500)). Rigorous bounds on $\beta_{M S T}$ and $\beta_{T S P}$ are still relatively crude, but for the TSP there have been increasingly sophisticated simulations with bounded errors on the TSP calculations. Record holders Johnson, McGeoch and Rothberg (1996) give

$$
\beta_{T S P}=0.7124 \pm 0.0002
$$

Moscato and Norman (1998) also give a fractal, spacefilling heuristic for which they determine the exact value $\beta_{M N * T S P}$ of their limit constant,

$$
\beta_{M N * T S P}=\frac{4(1+2 \sqrt{2}) \sqrt{51}}{153}=0.7147 \ldots
$$

This is certainly intriguing, but the spacefilling model and the independent uniform model are not perfect matches.

In a remarkable paper Avram and Bertsimas (1992) give an exact formula for $\beta_{M S T}$, but it comes at the price of an infinite sum of integrals that are not easy to evaluate. The authors required an integration tour de force for to give a rigorous proof (in their Theorem 9) that

$$
\beta_{M S T} \geq 0.0600822 .
$$

In a brief but interesting simulation study, Cortina-Borja and Robinson (2000) estimated that

$$
\beta_{M S T}=0.6331 \pm \widehat{s e} 0.0013
$$


This estimate is based on relatively small sample size (with $n$ not bigger than $2^{15}=32,768$ ) and, since fast algorithms are available for the MST, it seems feasible to extend the analysis to much larger sample sizes.

Returning to spanning caterpillars, we note that for any set $\chi=\left\{x_{1}, x_{2}, \ldots, x_{n}\right\}$, we have the elementary bounds

$$
\min (1, \lambda) M S T(\chi) \leq M S C_{\lambda}(\chi) \leq \max (1, \lambda) T S P(\chi),
$$

where $T S P(\chi)$ denotes the length of shortest Euclidean path through $\chi, M S T(\chi)$ denotes the Euclidean length of the minimal spanning tree, and $M S C_{\lambda}(\chi)$ is the weight of the minimum spanning caterpillar with path edge weight factor $\lambda$. These bounds are crude, but they do show that $\beta_{M S C}(\lambda)$ is strictly positive for all $\lambda>0$. For $\lambda=1$, the minimum spanning caterpillar constant $\beta_{M S C}(1)$ also inherits the suggestive simulation bounds (30) and (33) as well as any rigorous bounds that are proved for $\beta_{T S P}$ and $\beta_{M S T}$.

For exact optimum and for $(1+\epsilon)$ approximations, the computational complexity of the MSC is much closer to the notoriously hard TSP than to the notoriously easy MST. Even if some generous oracle were to identify the set of vertices that are on the path of an optimal MSC, one would still need to solve a traveling salesman problem to put those vertices into an optimal order. While this observation may fall short of a formal proof that the Euclidean MSC problem is NP-hard, it cannot not fall short by much.

The question of approximate solution of the Euclidean MSC problem is much more interesting. It seems inevitable that the approximation schemes of Arora (1998) can be modified to provide a solution of the Euclidean MSC problem that is within in a factor of $1+1 / c$ of the optimum and do so with a running time of $O\left(n\left(\log ^{O(C)}(n)\right)\right.$. We have not pursued this point, but it does seem worth pursuing.

Even though though our interest in minimum weight caterpillars comes mainly from communication networks, we should note that caterpillars have a long history in graph theory. Harary and Schwenk (1973) used Pólya enumeration theory to show that the number of non-isomorphic caterpillars on $n+4$ vertices is given by the elegant formula $2^{n}+2^{\lfloor n / 2\rfloor}$, and, in the same paper (p. 361), the authors credit A. Hobbs for introducing the term "caterpillar." Harary and Schwenk (1971) and Harary and Schwenk (1972) had earlier investigated the connectivity properties of graph powers of caterpillars. More recently, Ortiz and Villanueva (2012) studied independent sets in caterpillar graphs and found that the whole family of independent sets can be found in polynomial time. Caterpillars are the simplest graphs for which the graphical bandwidth problem is non-trivial, so in many bandwidth investigations caterpillars are important - see, e.g. Assmann, Peck, Sysło and Zak (1981), Miller (1981), Monien (1986), Haralambides, Makedon and Monien (1991), Feige and Talwar (2005), and Lin, Lin and Xu (2006).

Finally, we should note that in chemistry, caterpillars are also known as Gutman trees, the later name referring back to Gutman (1977); sometimes they are also called benzenoid trees because of their common presence in the structure of benzenoid hydrocarbons. Surveys of El-Basil (1987), El-Basil (1990) and El-Basil (2008) detail these connections and give many further references.

\section{Two Concluding Observations}

T. Tao's essay on the extended reals (Tao (2008, pp. 38-56)) makes the case that the extend real numbers ${ }^{*} \mathbb{R}$ can be of concrete benefit to almost any mathematician, pure or applied. Here with a very light use of the extended reals, we can answer a 
question that was raised in the introduction; we just need to take our path weight $\lambda$ to be a strictly positive infinitesimal.

Specifically, we fix $\lambda$ to be any extended real such that $0<\lambda<x$ for all $x \in \mathbb{R}$ with $x>0$. With this choice, the weight of the minimum spanning caterpillar is just $\lambda$ times the (usual Euclidean) cost of the optimal traveling salesman path. Thus, if one reformulates Theorem 1 to permit an infinitesimal $\lambda \in{ }^{*} \mathbb{R}$, we see that Theorem 1 is a strict generalization of the Beardwood, Halton, Hammersley theorem. Moreover, a proof of Theorem 1 that allows for a strictly positive infinitesimal $\lambda \in{ }^{*} \mathbb{R}$ is virtually identical to the proof we have given here.

It is fair to say that this (very light) use of the extend reals is just a mater of language. Nevertheless, we were genuinely uncertain at one point if one could view Theorem 1 as an honest generalization of the Beardwood, Halton, Hammersley theorem. It eventually became clear that the formal introduction of the extended reals would make an affirmative answer easy. While this may be a just matter of language, at the end of the day, it seems to be an instance of useful language.

Our second observation also concerns the flexibility of the free parameter $\lambda$. This parameter was introduced because of modeling motivations of the kind that were mentioned in the introduction. Nevertheless, after $\lambda$ enters the game, it presents new mathematical possibilities - possibilities that are not present in problems like

the TSP and MST. For example, $\beta_{M S C}(\lambda)$ is differentiable, and $\beta_{M S C}^{\prime}(\lambda)$ provides a measure of the relative weight that is placed on the path edges in the limit. Thus, the free parameter $\lambda$ offers a special handle on the asymptotic geometry of the MSC for which there are no direct analogs in the traditional theory of the TSP or MST.

\section{REFERENCES}

Arora, S. (1998), 'Polynomial time approximation schemes for Euclidean traveling salesman and other geometric problems', J. ACM 45(5), 753-782.

Assmann, S. F., Peck, G. W., Sysło, M. M. and Zak, J. (1981), 'The bandwidth of caterpillars with hairs of length 1 and 2', SIAM J. Algebraic Discrete Methods 2(4), 387-393.

Avram, F. and Bertsimas, D. (1992), 'The minimum spanning tree constant in geometrical probability and under the independent model: a unified approach', Ann. Appl. Probab. 2(1), 113130.

Beardwood, J., Halton, J. H. and Hammersley, J. M. (1959), 'The shortest path through many points', Proc. Cambridge Philos. Soc. 55, 299-327.

Boucheron, S., Lugosi, G. and Bousquet, O. (2004), Concentration inequalities, in O. Bousquet, U. von Luxburg and G. Rätsch, eds, 'Advanced Lectures on Machine Learning', Vol. 3176 of Lecture Notes in Computer Science, Springer Berlin / Heidelberg, pp. 208-240.

Cortina-Borja, M. and Robinson, T. (2000), 'Estimating the asymptotic constants of the total length of Euclidean minimal spanning trees with power-weighted edges', Statist. Probab. Lett. 47(2), 125-128.

El-Basil, S. (1987), 'Applications of caterpillar trees in chemistry and physics', Journal of Mathematical Chemistry 1, 153-174.

El-Basil, S. (1990), Caterpillar (gutman) trees in chemical graph theory, in I. Gutman and S. Cyvin, eds, 'Advances in the Theory of Benzenoid Hydrocarbons', Vol. 153 of Topics in Current Chemistry, Springer Berlin / Heidelberg, pp. 273-289.

El-Basil, S. (2008), 'Combinatorial Properties of Graphs and Groups of Physico-Chemical Interest', Combinatorial Chemistry \& High Throughput Screening 11(9), 707-722.

Feige, U. and Talwar, K. (2005), Approximating the bandwidth of caterpillars, in 'Approximation, randomization and combinatorial optimization', Vol. 3624 of Lecture Notes in Comput. Sci., Springer, Berlin, pp. 62-73.

Few, L. (1955), 'The shortest path and the shortest road through n points in a region', Mathematika 2, 141-144. 
Finch, S. R. (2003), Mathematical constants, Vol. 94 of Encyclopedia of Mathematics and its Applications, Cambridge University Press, Cambridge.

Gutman, I. (1977), 'Topological properties of benzenoid systems', Theoretical Chemistry Accounts: Theory, Computation, and Modeling (Theoretica Chimica Acta) 45, 309-315.

Haralambides, J., Makedon, F. and Monien, B. (1991), 'Bandwidth minimization: an approximation algorithm for caterpillars', Math. Systems Theory 24(3), 169-177.

Harary, F. and Schwenk, A. (1971), 'Trees with hamiltonian square', Mathematika 18, 138-140.

Harary, F. and Schwenk, A. (1972), 'A new crossing number for bipartite graphs', Utilitas Math. 1, 203-209.

Harary, F. and Schwenk, A. J. (1973), 'The number of caterpillars', Discrete Math. 6, 359-365.

Johnson, D. S., McGeoch, L. A. and Rothberg, E. E. (1996), Asymptotic experimental analysis for the Held-Karp traveling salesman bound, in 'Proceedings of the Seventh Annual ACM-SIAM Symposium on Discrete Algorithms (Atlanta, GA, 1996)', ACM, New York, pp. 341-350.

Korevaar, J. (2004), Tauberian theory, Vol. 329 of Grundlehren der Mathematischen Wissenschaften [Fundamental Principles of Mathematical Sciences], Springer-Verlag, Berlin. A century of developments.

Lin, M., Lin, Z. and Xu, J. (2006), 'Graph bandwidth of weighted caterpillars', Theoret. Comput. Sci. 363(3), 266-277.

Lindvall, T. (1992), Lectures on the coupling method, Dover Publications.

Miller, Z. (1981), The bandwidth of caterpillar graphs, in 'Proceedings of the Twelfth Southeastern Conference on Combinatorics, Graph Theory and Computing, Vol. II (Baton Rouge, La., 1981)', Vol. 33, pp. 235-252.

Monien, B. (1986), 'The bandwidth minimization problem for caterpillars with hair length 3 is NP-complete', SIAM J. Algebraic Discrete Methods 7(4), 505-512.

Moscato, P. and Norman, M. G. (1998), 'On the performance of heuristics on finite and infinite fractal instances of the Euclidean traveling salesman problem', INFORMS J. Comput. 10(2), 121-132.

Ortiz, C. and Villanueva, M. (2012), 'Maximal independent sets in caterpillar graphs', Discrete Appl. Math. 160(3), 259-266.

Steele, J. M. (1986), 'An Efron-Stein inequality for nonsymmetric statistics', Ann. Statist. 14(2), 753-758.

Steele, J. M. (1997), Probability theory and combinatorial optimization, Vol. 69 of CBMS-NSF Regional Conference Series in Applied Mathematics, Society for Industrial and Applied Mathematics (SIAM), Philadelphia, PA.

Tao, T. (2008), Structure and randomness, American Mathematical Society, Providence, RI. Pages from year one of a mathematical blog.

Yukich, J. E. (1998), Probability theory of classical Euclidean optimization problems, Vol. 1675 of Lecture Notes in Mathematics, Springer-Verlag, Berlin. 